\title{
First Report on Megaselia scalaris Loew (Diptera: Phoridae) Infestation of the Invasive Pest Spodoptera frugiperda Smith (Lepidoptera: Noctuidae) in China
}

\author{
Yunlin Tang 1,2,3, Qingyan Li 1,2,3, Li Xiang 1,4, Ruocheng Gu 1,2,3, Yanyan Wu 1,2,3, Yonghong Zhang 5 , \\ Xingrong Bai ${ }^{5}$, Xiaohui Niu ${ }^{6}$, Tian Li ${ }^{1,2,3}{ }^{(D)}$, Junhong Wei ${ }^{1,2,3, *}$, Guoqing Pan 1,2,3,* and Zeyang Zhou ${ }^{1,2,3,7}$ \\ 1 State Key Laboratory of Silkworm Genome Biology, Southwest University, Chongqing 400715, China; \\ t7anthony@email.swu.edu.cn (Y.T.); Li_qingyann@163.com (Q.L.); xiang163li@163.com (L.X.); \\ guruocheng96@163.com (R.G.); wyy0711@email.swu.edu.cn (Y.W.); lit@swu.edu.cn (T.L.); \\ zyzhou@swu.edu.cn (Z.Z.) \\ 2 Chongqing Key Laboratory of Microsporidia Infection and Control, Southwest University, \\ Chongqing 400715, China \\ 3 Key Laboratory of Sericultural Biology and Genetic Breeding, Ministry of Agriculture, Southwest University, \\ Chongqing 400715, China \\ 4 School of Biotechnology, Southwest University, Chongqing 400715, China \\ 5 Institute of Sericulture and Apiculture, Yunnan Academy of Agriculture Sciences, Mengzi 661101, China; \\ zhang200503@126.com (Y.Z.); bxrong3@163.com (X.B.) \\ 6 Crop Seed Management Station of Chongqing, Chongqing 401121, China; xiaohui749@126.com \\ 7 College of Life Sciences, Chongqing Normal University, Chongqing 401331, China \\ * Correspondence: weijunhong@swu.edu.cn (J.W.); gqpan@swu.edu.cn (G.P.)
}

check for updates

Citation: Tang, Y.; Li, Q.; Xiang, L.; Gu, R.; Wu, Y.; Zhang, Y.; Bai, X.; Niu, X.; Li, T.; Wei, J.; et al. First Report on Megaselia scalaris Loew (Diptera:

Phoridae) Infestation of the Invasive Pest Spodoptera frugiperda Smith (Lepidoptera: Noctuidae) in China. Insects 2021, 12, 65. https://doi.org/ $10.3390 /$ insects 12010065

Received: 3 December 2020 Accepted: 8 January 2021 Published: 13 January 2021

Publisher's Note: MDPI stays neutral with regard to jurisdictional clai$\mathrm{ms}$ in published maps and institutional affiliations.

Copyright: (C) 2021 by the authors. Licensee MDPI, Basel, Switzerland. This article is an open access article distributed under the terms and conditions of the Creative Commons Attribution (CC BY) license (https:// creativecommons.org/licenses/by/ $4.0 /)$
Simple Summary: The invasive pest Spodoptera frugiperda first emerged in China in 2019, and therefore the information on indigenous natural enemies of $S$. frugiperda has been limited in China. In this study, we reported that a dipteran species was observed to infest $S$. frugiperda collected from maize fields in four different regions of China. Further morphological and molecular recognition identified all the flies as Megaselia scalaris. The findings of this study will improve our understanding on natural enemies to $S$. frugiperda and potentially provide new ideas for integrated pest management strategies in China.

Abstract: The invasive pest Spodoptera frugiperda first emerged in China in January 2019 and has, to date, migrated to 29 provinces and municipalities in China, causing heavy crop damage in large areas. As a response to this invasive species from the environment, some indigenous natural enemies have been discovered and reported after $S$. frugiperda invasion. In this paper, parasitic flies were collected and identified from S. frugiperda collected in the Yunnan, Guangxi, and Henan provinces and the Chongqing municipality in China. By using both conventional and molecular approaches, we were able to show that all the parasitic flies of $S$. frugiperda identified in the four regions were Megaselia. scalaris, and that they attacked the pest larvae and pupae. This is the first report on an indigenous Chinese Megaselia species that has parasitic ability against the invasive pest $S$. frugiperda, potentially providing new ideas for pest control in China.

Keywords: Spodoptera frugiperda; invasive pest; natural enemies; Megaselia scalaris; pest management

\section{Introduction}

As a major migratory agricultural pest, Spodoptera frugiperda originated in the Americas and migrated to the African continent in 2016. It was first discovered and reported in China in January 2019 [1,2]. With favorable temperatures and abundant crops, S. frugiperda rapidly spread to 29 provinces (autonomous regions, municipalities) across the country, posing a serious threat to food production security in China [3]. To control this new invasive and 
rapidly spreading pest, the "2019 Spodoptera frugiperda prevention and control technology plan (trial)" developed by Ministry of Agriculture and Rural Affairs of the People's Republic of China recommended employing the chemical pesticides chlorantraniliprole, cyfluthrin, and deltamethrin for emergency control. In the following years, the pest may continue to cause seasonal outbreaks in China. The climate in southern China can support the presence $S$. frugiperda through the winter and this region could potentially be the annual breeding area for the pest [3]. In addition to emergency control by chemical pesticides, there is an urgent need for a long-term integrated pest management (IPM) strategy for S. frugiperda control. Biological control and natural enemy protection and utilization should be given priority, in addition to efforts towards reducing the number of applications and risks of pesticides [4].

Many kinds of potentially natural candidates for biological control, including entomophagous insects and entomopathogens, have been identified and reported throughout the world [5]. Investigations conducted in the Americas on the inventory of parasitoids and parasites of $S$. frugiperda have shown that approximately 150 species from 14 families are able to attack S. frugiperda [6,7]. Moreover, virus-based insecticides such as $S$. frugiperda multiple nucleopolyhedrovirus ( $S f \mathrm{MNPV}$ ) and granulovirus $(S f \mathrm{GV})$ have been developed for $S$. frugiperda [8,9]. S. frugiperda has exhibited resistance to many commonly used entomopathogenic bacteria and fungi such as Bacillus thuringiensis and Beauveria bassiana $[10,11]$. The information on indigenous natural enemies of $S$. frugiperda has been limited in China because this species of pest only recently migrated to China (2019). Within the past year, several surveys on the natural enemies attacking S. frugiperda have been conducted in many regions of China, showing that a number of parasitic wasps and flies are able to attack S. frugiperda. These include Telenomus remus, Diadegma semiclausum, and Exorista japonica $[12,13]$.

In this study, we serendipitously found that $S$. frugiperda was parasitized by some flies from four regions in China, and identified these flies as being of the species Megaselia scalaris by DNA barcoding $[14,15]$. This is the first report showing that $M$. scalaris could parasitize S. frugiperda in China. Our discovery expands the repertoire of indigenous natural enemies against $S$. frugiperda, and can ultimately contribute to the development of IPM strategies for pest control in China.

\section{Materials and Methods}

\subsection{Parasitic Fly Collections}

In this study, five groups (YN, GX, CQ_2, HN, and CQ_1) of parasitic flies infesting S. frugiperda were collected from four regions in China (Figure 1). Of these, four were found in containers of $S$. frugiperda collected in Yunnan (YN), Guangxi (GX), Chongqing (CQ_2), and Henan $(\mathrm{HN})$. The remaining group was found in the culture medium for $S$. frugiperda intestinal fungi isolation and was designated as CQ_1. Flies were captured with a combination of sweep netting over the container of $S$. frugiperda and maggots were collected into tubes directly. For each group 30-50 flies were collected, and 24 maggots were collected for the CQ_1 group. Following collection, flies and maggots were anesthetized over ice.

\subsection{Scanning Electron Microscopy (SEM)}

The anesthetized flies were washed by sterilized phosphate buffer saline (PBS) and preserved in $75 \%$ ethanol for $24 \mathrm{~h}$. They were then put in a critical point-drier and fixated with double-side adhesive tape, and then coated with gold in vacuum condition. Subsequently, electron microscopy observations were conducted to observe details with regard to the mouthpart, compound eyes, wing, and terminalia for morphological identification with a Hitachi SU3500 Scanning Electron Microscope.

\subsection{DNA Extraction and Gene Amplification}

The CTAB method was employed to extract genomic and mitochondrial DNA of parasitic flies [16]. Two pairs of DNA barcode primers were used to amplify the 28S rRNA gene 
(F: 5'-GACTACCCCCTGAATTTAAGCAT-3' and R: 5'-GACTCCTTGGTCCGTGTTTCAAG$3^{\prime}$ ) and cytochrome oxidase subunit I (COI) (F: 5'-GGTCAACAAATCATAAAGATATTGG$3^{\prime}$ and R: 5'-TAAACTTCAGGGTGACCAAAAAATCA-3') gene of flies [14,15]. The PCR program was as follows: $96{ }^{\circ} \mathrm{C}$ for $8 \mathrm{~min}$; 35 cycles at $96{ }^{\circ} \mathrm{C}$ for $40 \mathrm{~s} ; 56{ }^{\circ} \mathrm{C}$ for $30 \mathrm{~s}$; $72{ }^{\circ} \mathrm{C}$ for $1 \mathrm{~min}$; and then $72{ }^{\circ} \mathrm{C}$ for $10 \mathrm{~min}$. The specific PCR products were then assessed by $1.5 \%$ agarose gel electrophoresis and subsequently sequenced by Sangon Biotech (Shanghai, China) Co., Ltd. All sequences of the targeted genes in this study were submitted to NCBI under the accession number MT254753-MT254757 (28S rRNA), MT251290MT251294 (COI).

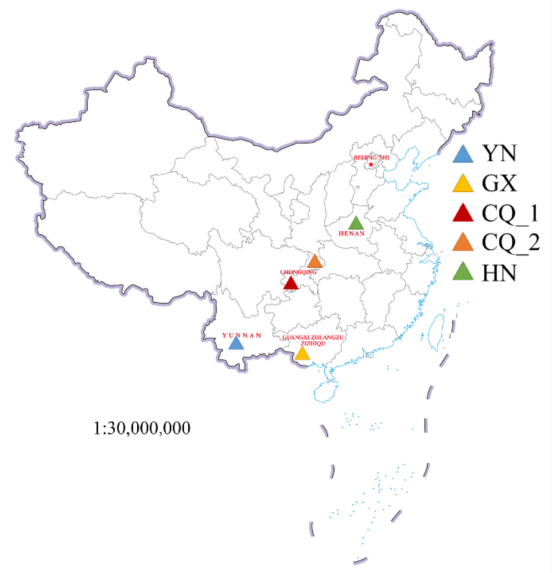

Figure 1. Sampling sites of parasitic flies and Spodoptera. frugiperda in China (Yunnan (YN), Guangxi (GX), Chongqing (CQ_1, CQ-2), and Henan (HN)).

\subsection{Data Analyses}

The sequences of the 28S rRNA and COI gene of S. frugiperda were used to blast in the NCBI database. The top 100 sequences of each gene were downloaded and redundant low-quality sequences were removed after being aligned by MUSCLE v3.8.31 [17]. Phylogenetic trees were constructed using the neighbor-joining (NJ) method in MEGA5 [18].

\section{Results}

In our surveillance studies, some flies $(2.46 \pm 0.50$ by $0.75 \pm 0.17 \mathrm{~mm})$ were observed and caught in the closed but ventilated containers for $S$. frugiperda collected from the maize fields of four different regions in China (Figure 2A), and white long oval eggs $(0.60 \pm 0.04$ by $0.2 \pm 0.01 \mathrm{~mm}$ ) were found on the epidermis of $S$. frugiperda larvae (Figure $2 B, C$ ) and pupae (Figure 2D). Moreover, we observed that the maggots emerged from thoracic cavities of $S$. frugiperda pupae, which were alive upon being collected from the maize fields, and left solid exoskeletons of $S$. frugiperda pupae as remnants (Figure 2E). Additionally, the maggots that emerged on the solid fungi culture medium (Potato Dextrose Agar Medium, PDA) were inoculated with the intestinal tract portion of $S$. frugiperda larvae (Figure 2F). To better characterize these flies morphologically, we next conducted a scanning electron microscopy (SEM) investigation (Figure 3), and concluded that these flies were of the species M. scalaris based on the presence of male terminalia from the SEM results (Figure 3J) [19]. To further confirm the identification of these flies by molecular approach, two pairs of universal DNA barcode primers were used to amplify the target genes from nuclear DNA and mitochondrial DNA of flies. The sequence alignments showed that all the parasite flies caught in different regions shared high homology in two DNA barcoding genes. The phylogenetic trees (Figure 4) constructed based on the 28S rRNA (Figure 4A) and $\mathrm{COI}$ (Figure $4 \mathrm{~B}$ ) gene sequence show that the five fly specimens from this study have a high homologous evolutionary relationship with the Phoridae family, and clustered with M. scalaris. 


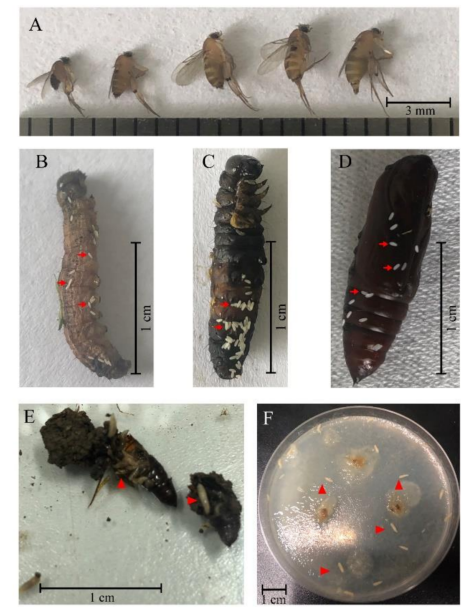

Figure 2. Megaselia scalaris specimens caught in S. frugiperda containers (A), S. frugiperda specimens with several eggs of M. scalaris (B-D), M. scalaris larvae emerging from the $S$. frugiperda pupa (E), and the medium for $S$. frugiperda intestinal fungi isolation (F). (A-E) were from Yunnan, and (F) originated in Chongqing. Red arrows indicate the eggs of M. scalaris, red triangles indicate the M. scalaris larvae.
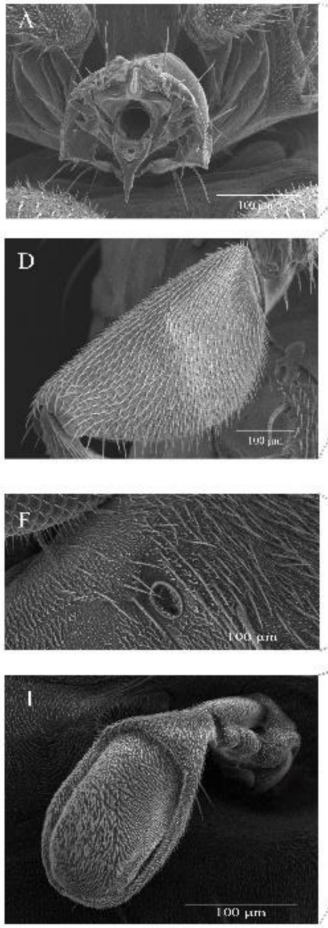
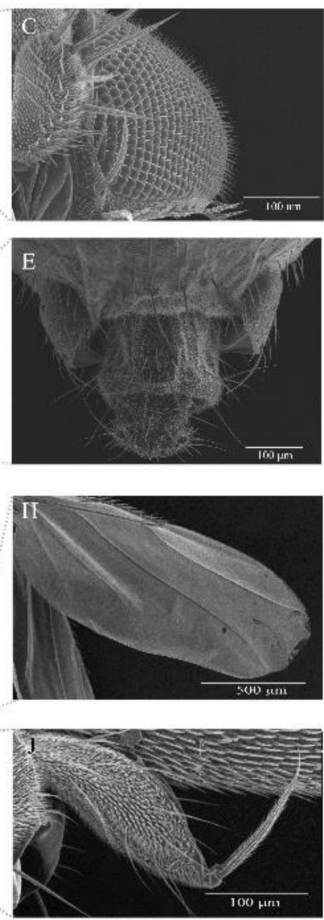

Figure 3. Scanning electron micrograph of the parasitic flies $M$. scalaris from Chongqing (female: (A-E), male: (F-J)). Female: (A) mouthpart, (B) segmental venter of female, (C) compound eyes, (D) tibia, and (E) female terminalia. Male: (F) spiracle, (G) left face of male, (H) wing, (I) haltere, and $(\mathbf{J})$ male terminalia. 
$\mathbf{A}$

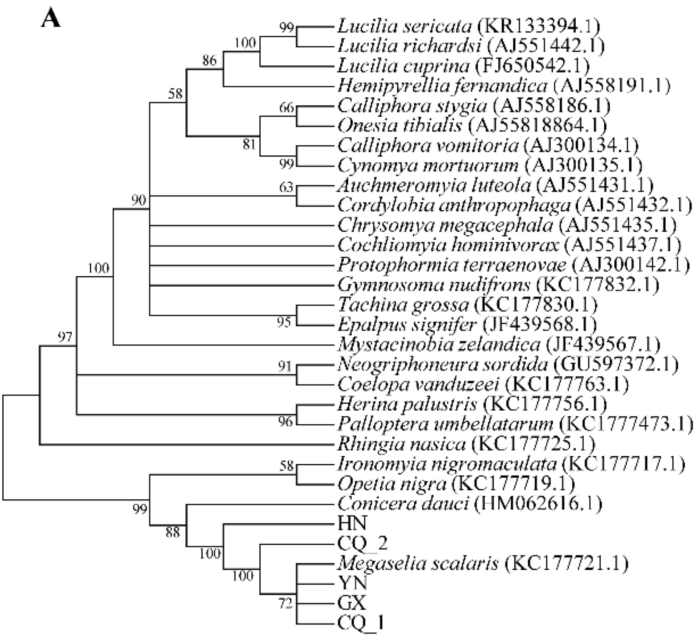

B

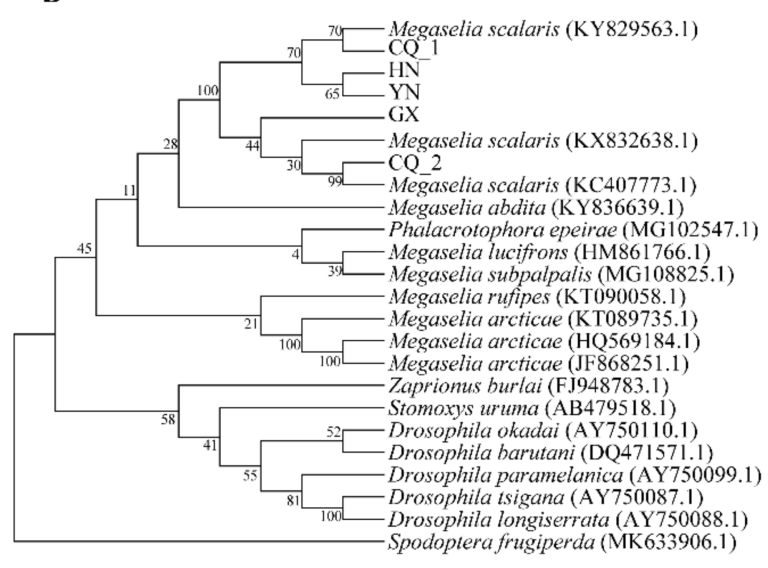

Figure 4. Phylogenetic tree of two DNA barcode sequences ((A) 28S rRNA, (B) COI) of five M. scalaris specimens and comparative species.

\section{Discussion}

Since S. frugiperda invaded China, Chinese researchers have conducted investigations to identify natural enemies that are able to parasitize $S$. frugiperda. Egg parasitoid wasps $[20,21]$ and some other natural enemies parasitizing $S$. frugiperda larvae were found and reported [12,13]. In this study, some dipterans infesting S. frugiperda were observed and caught in four regions of China. Morphological and DNA barcode identification confirmed that all the fly specimens collected in the four regions were of the species of $M$. scalaris.

$M$. scalaris is active in a wide range of geographical regions and usually feeds on decaying organic materials [22]. It has been reported in forensic cases [23], and there are some reports on the parasitism of $M$. scalaris. It has been reported that adult $M$. scalaris can break into the hive and lay eggs in the hive; the larvae of $M$. scalaris then hijack the food of bee larvae, therefore hindering the growth and development of bee larvae [24]. Koch and Costa found that laboratory-raised Parastagmatoptera tessellata and Triatoma brasiliensis could be parasitized by $M$. scalaris [25,26]; the larvae of $M$. scalaris can feed and grow in the host's body. In our study, a similar phenomenon of $M$. scalaris parasitizing $S$. frugiperda in various regions of China was observed. The emergence of eggs on the epidermis of $S$. frugiperda larvae and pupae (Figure 2B-D), and maggots in the thoracic cavities of $S$. frugiperda pupae (Figure 2E) and the solid fungi culture medium inoculated with the intestinal tract portion of $S$. frugiperda larvae (Figure $2 \mathrm{~F}$ ) demonstrates that maggots can destroy the integrity of the epidermis and penetrate into the host body cavity to infest $S$. frugiperda. The emergence of flies (Figure 2A) in the closed but ventilated containers proves that the maggots are able to develop to the adult stage in the body cavity of $S$. frugiperda. All these results indicate that the indigenous species $M$. scalaris can parasitize the new invasive pest $S$. frugiperda in the wild in China. To our knowledge, this is the first record of $M$. scalaris infesting wild S. frugiperda in China. There have been a few previous reports of this fly infesting other insects in the wild [24,27-29]. Our finding indicates that $M$. scalaris may play various roles in the ecosystem in addition to being saprophages, such as being parasitic to insects.

In consideration of biological control to $S$. frugiperda, several native Chinese insects are reported to be parasitic and predatory to the new invasive pest $S$. frugiperda. However, their potential applications as biological control agents remain unclear and deserve further investigation. In terms of developing effective IPM strategies for controlling S. frugiperda in China, biological control, protection, and utilization of natural enemies should be given priority for reducing the use of pesticides. Therefore, there needs to be further investigation and exploitation of natural enemies in China to aid the development of subsequent IPM strategies. 


\section{Conclusions}

In this study, we reported that some dipterans were able to infest invasive pest S. frugiperda in the wild in China. Both conventional and molecular methods identified the dipterans as M. scalaris. This is the first report on the native species $M$. species infesting S. frugiperda in China. Further investigations on the infestation of S. frugiperda by M. scalaris are warranted and such studies can potentially provide new directions for the integrated pest management strategies in China.

Author Contributions: Conceptualization, T.L., J.W., G.P., and Z.Z.; methodology, validation, and formal analysis; investigation, T.L., Q.L., L.X., R.G., and Y.W.; resources, T.L., Y.Z., X.B., and X.N.; writing — original draft preparation, Y.T.; writing — review and editing, G.P. and J.W.; All authors have read and agreed to the published version of the manuscript.

Funding: This research was funded by the Fundamental Research Funds for Central Universities (XDJK2018AA001 and XDJK2019C010) and the Natural Science Foundation of Chongqing, China (cstc2019jcyj-msxmX0511).

Institutional Review Board Statement: Not applicable.

Informed Consent Statement: Not applicable.

Data Availability Statement: All the data generated in this study have been submitted to NCBI for public availability under the accession number MT254753-MT254757 (28S rRNA), MT251290MT251294 (CO I).

Acknowledgments: We thank Zhu Feng from Zaozhuang University and Huang Xuhua from General Station of Sericulture Technology Popularization of Guangxi for resource support.

Conflicts of Interest: The authors declare no conflict of interest.

\section{References}

1. Georg, G.P.; Lava, K.; Sagnia, B.S.; Abou, T.; Manuele, T. First Report of Outbreaks of the Fall Armyworm Spodoptera frugiperda (J E Smith) (Lepidoptera, Noctuidae), a New Alien Invasive Pest in West and Central Africa. PLoS ONE 2016, 11, e0165632.

2. Wu, Q.-L.; Jiang, Y.-Y.; Wu, K.-M. Analysis of Migration Routes of the Fall Armyworm Spodoptera frugiperda (J.E. Smith) from Myanmar to China. Plant Prot. 2019, 45, 1-6.

3. Jiang, Y.-Y.; Liu, J.; Xie, M.-C.; Li, Y.-H.; Yang, J.-J.; Zhang, M.-L.; Qiu, K. Observation on Law of Diffusion Damage of Spodoptera frugiperda in China in 2019. Plant Prot. 2019, 45, 10-19.

4. Birhanu, S.; Josephine, S.; Peter, M.; Paddy, L.; Esayas, M.; Nsami, E.; Mulatu, W.; Gashawbeza, A.; Tadele, T. First Report of the Fall Armyworm, Spodoptera frugiperda (Lepidoptera: Noctuidae), Natural Enemies from Africa. J. Appl. Entomol. 2018, $142,800-804$.

5. Koffi, D.; Kyerematen, R.; Eziah, V.; Agboka, K.; Adom, M.; Goergen, G.; Meagher, R. Natural Enemies of the Fall Armyworm, Spodoptera frugiperda (J.E. Smith) (Lepidoptera: Noctuidae) in Ghana. Fla. Entomol. 2020, 103, 85-90. [CrossRef]

6. Jaime, M.-O.; Roberto, L.-G.; Martin, G.-R.; Marilu, L.-E.; Manue, A.R.-V.; Francisco, A.-P. Pathogens and Parasitic Nematodes Associated with Populations of Fall Armyworm (Lepidoptera: Noctuidae) Larvae in Mexico. Fla. Entomol. 2003, 86, 244-253.

7. Jaime, M.-O.; James, C.; Elvis, H.; John, F. Parasitoids and Parasites of Spodoptera frugiperda (Lepidoptera: Noctuidae) in the Americas and Caribbean Basin: An inventory. Fla. Entomol. 2003, 86, 254-289.

8. Robert, B.; Holly, P. Laboratory and Field Evaluations of the Efficacy of a Fast-killing Baculovirus Isolate from Spodoptera frugiperda. J. Invertebr. Pathol. 2012, 109, 194-200.

9. Santiago, H.; Alicia, S.-C.; Víctor, R. Baculovirus Insecticides in Latin America: Historical Overview, Current Status and Future Perspectives. Viruses 2015, 7, 2230-2267.

10. Wraight, S.P.; Ramos, M.E.; Avery, P.B.; Jaronski, S.T.; Vandenberg, J.D. Comparative Virulence of Beauveria Bassiana Isolates Against Lepidopteran Pests of Vegetable Crops. J. Invertebr. Pathol. 2010, 103, 186-199. [CrossRef]

11. Ricardo, A.P.; Silva, R.F.P.d.; Lidia, M.F. Effectiveness of Bacillus Thuringiensis Strains Against Spodoptera frugiperda (Lepidoptera: Noctuidae). Braz. J. Microbiol. 2000, 31, 165-167.

12. Ning, S.-F.; Zhou, J.-C.; Zhang, Z.-T.; Dong, Q.-J.; Bai, X.-P.; Zhang, L.-S.; Dong, H. Five Parasitic Natural Enemies of Spodoptera frugiperda and Two Hyperparasitoids of Cotesia Glomerata Were Found in Southeast of Guizhou Province. Plant Prot. 2019, 45, 39-42.

13. Zhang, Z.-T.; Qin, S.-Z.; Li, J.; Wang, Y.; Sun, X.; Dong, H.; Liu, J.-W.; Song, X.-Y. Preliminary Investigation of Parasitic Natural Enemies Against Spodoptera frugiperda (J. E. Smith) in Southeast of Guizhou Province. Plant Prot. 2019, 45, 65-69.

14. Hiroshi, Y.; Shinta, F. Two New Species in the Echinoderes coulli Group (Echinoderidae, Cyclorhagida, Kinorhyncha) from the Ryukyu Islands, Japan. ZooKeys 2014, 382, 27-52. 
15. Folmer, O.; Black, M.; Hoeh, W.; Lutz, R.; Vrijenhoek, R. DNA Primers for Amplification of Mitochondrial Cytochrome C Oxidase Subunit I from Diverse Metazoan Invertebrates. Mol. Mar. Biol. Biotechnol. 1994, 3, 294-299.

16. Arseneau, J.-R.; Steeves, R.; Mark, L. Modified Low-salt CTAB Extraction of High-quality DNA from Contaminant Rich Tissues. Mol. Ecol. Resour. 2016, 17, 686-693. [CrossRef]

17. Edgar, R. MUSCLE: Multiple Sequence Alignment with High Accuracy and High Throughput. Nucleic Acids Res. 2004, 32, 1792-1797. [CrossRef]

18. Koichiro, T.; Daniel, P.; Nicholas, P.; Glen, S.; Masatoshi, N.; Sudhir, K. MEGA5: Molecular Evolutionary Genetics Analysis Using Maximum Likelihood, Evolutionary Distance, And Maximum Parsimony Methods. Mol. Biol. Evol. 2011, 28, $2731-2739$.

19. Disney, R.; Aguiar, A. Scuttle Flies (Diptera: Phoridae) of Madeira. Fragm. Faun. 2008, 51, 23-62. [CrossRef]

20. Li, F.; Wang, L.; Lv, B.; Cao, F.; Pan, X.; Yuan, L.; Wu, S. The Report of Chelonus munakatae Parasitizing Fall Armworm Spodoptera frugiperda (Lepidoptera: Noctuidae) in Hainan, China. Chin. J. Biol. Control 2019, 35, 992-996.

21. Li, Z.; Lv, X.; Ya, Y.; Xu, Q.; Ye, J.; Han, S.; Zhang, C.; Li, J. The Parasitism of Telenomus remus and Trichogramma chilonis on Spodoptera frugiperda Found in the Fields of Guangzhou and Hong Kong. J. Environ. Entomol. 2019, 41, 760-765.

22. Disney, R. Natural history of the Scuttle fly, Megaselia scalaris. Annu. Rev. Entomol. 2008, 53, 39-60. [CrossRef] [PubMed]

23. Bernard, G.; Jeffrey, W. Forensic Use of Megaselia abdita and M. scalaris (Phoridae: Diptera): Case Studies, Development Rates, and Egg Structure. J. Med. Entomol. 1998, 35, 205-209.

24. Ahmed, H.M.; Niels, P.; Ivan, M.; Wahida, L.-A. Large Pathogen Screening Reveals First Report of Megaselia scalaris (Diptera: Phoridae) Parasitizing Apis mellifera Intermissa (Hymenoptera: Apidae). J. Invertebr. Pathol. 2016, 137, 33-37. [CrossRef]

25. Koch, M.; Fontanarrosa, P.; Padró, J.; Soto, I. First Record of Megaselia scalaris (Loew) (Diptera: Phoridae) Infesting Laboratory Stocks of Mantids (Parastagmatoptera tessellata, Saussure). Arthropods 2013, 2, 1-6.

26. Costa, J.; Almeida, C.; Esperança, G.; Morales, N.; Mallet, J.; Gonçalves, T.; Prado, A. First Record of Megaselia scalaris (Loew) (Diptera: Phoridae) Infesting Laboratory Colonies of Triatoma brasiliensis Neiva (Hemiptera: Reduviidae). Neotrop. Entomol. 2007, 36, 987-989. [CrossRef]

27. Sanguansub, S.; Siriyasatien, P.; Phumee, A.; Sunantaraporn, S.; Noknoy, R. Parasitism of Soldiers of the Termite, Macrotermes gilvus (Hagen), by the Scuttle Fly, Megaselia scalaris (Loew) (Diptera: Phoridae). Insects 2020, 11, 318. [CrossRef]

28. Sabo, R.; Legáth, J.; Staron, M.; Sabová, L. The First Record of Facultative Parasitism of Megaselia Spp. (Diptera: Phoridae) in a Honeybee Colony in Slovakia. Folia Vet. 2020, 64, 44-48. [CrossRef]

29. Debnath, P.; Roy, D. First Record of Megaselia scalaris (Loew) as a Potential Facultative Parasitoid of Apis mellifera in India. Asian J. Biol. 2019, 7, 1-9. [CrossRef] 